\title{
Collaboration in the competitive world of science: lessons to be learned from William T. Keeton
}

\author{
Günther K. H. Zupanc ${ }^{1}$
}

Received: 15 April 2015 / Revised: 21 April 2015 / Accepted: 24 April 2015 / Published online: 10 July 2015

(C) Springer-Verlag Berlin Heidelberg 2015

\begin{abstract}
The tremendous increase in the number of authors and institutional affiliations on papers published in the natural sciences over the last few decades is commonly interpreted as an indicator of an increase in the collaborative spirit. However, a closer analysis suggests that this development reflects an increase in cooperation (defined as a strategy to divide labor among participants), rather than a rise in collaboration (defined as a mutual engagement of participants in a coordinated effort to jointly solve a problem). An exception to this development was William T. Keeton (1933-1980), who, as a faculty member at Cornell University, pioneered research into pigeon homing. A direct result of his willingness to openly share ideas and collaborate with other investigators is the article by Hagstrum and Manley (J Comp Physiol A, 2015) in this issue of the Journal of Comparative Physiology A. Their study is based on data from experiments Keeton and his collaborators conducted some 40 years ago. Despite the age of these data, their analysis and the interpretation of the results are likely to stimulate fruitful discussion in the field of avian orientation.
\end{abstract}

Keywords Collaboration - Cooperation · Pigeon homing · Olfactory hypothesis $\cdot$ William T. Keeton

Günther K. H. Zupanc

g.zupanc@neu.edu

1 Laboratory of Neurobiology, Department of Biology, Northeastern University, 360 Huntington Avenue, Boston, MA 02115, USA

\section{Collaborations on the rise-or not?}

Research, particularly in the natural sciences, is increasingly carried out by teams, rather than by single investigators. Neurobiology, sensory physiology, and neuroethology - areas represented by the articles published in the Journal of Comparative Physiology A-are no exception to this trend. Between 1924 (the year the Journal was founded) and the mid-1960s, the vast majority of the papers were published by single authors. Since the 1970s, however, the number of authors per article has gradually increased to reach an average of 3.3 in 2014 (Fig. 1). This increase has been paralleled by a continuous decrease in the relative number of single-author papers. In 2014, only 5 out of a total of 82 original research and review articles were published by single authors. Similarly, until the 1990s the majority of studies published in the Journal of Comparative Physiology A were conducted in just one institution, whereas in the new millennium two institutional affiliations have become the norm (data not shown).

The results of this analysis seemingly confirm what has become a widely accepted notion: the number of collaborations has grown tremendously over the past few decades. However, does this trend reflect, as the numbers suggest, an increase in the collaborative spirit?

\section{Collaboration or cooperation?}

To answer this question, it might be useful to reflect on the difference between collaboration and what is commonly used as a synonym, cooperation. In learning science, collaboration is often defined as "a coordinated, synchronous activity that is the result of a continued attempt to 
Fig. 1 Mean number of authors per paper and percentage of single-author articles relative to the total number of articles, published in the Journal of Comparative Physiology A between 1924 and 2014. Over this period, all original research papers and review articles that appeared in a given year were analyzed at 10-year intervals, resulting in a total of 854 publications. Note that between 1944 and 1947 the publication of the Journal was temporarily interrupted

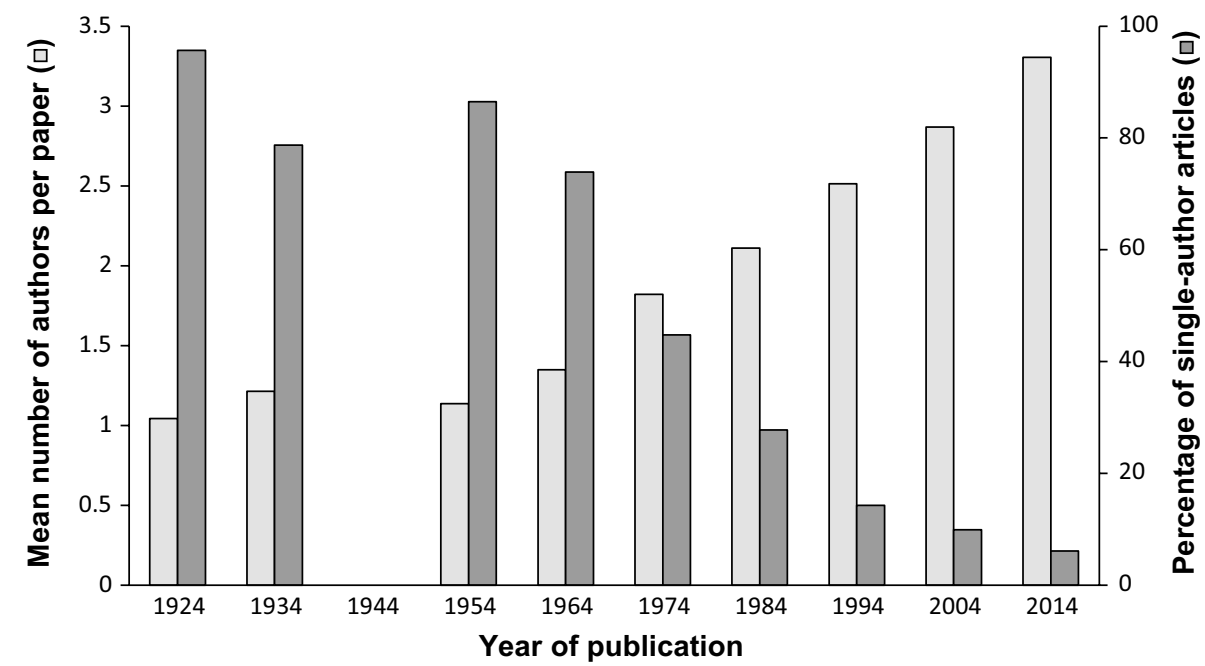

construct and maintain a shared conception of a problem", whereas "cooperative work is accomplished by the division of labor among participants, as an activity where each person is responsible for a portion of the problem solving" (Roschelle and Teasley 1995; p. 70). Randy Nelson of Pixar Animation Studios, a company with a reputation of collaborative spirit, put it more bluntly: "Cooperation... is a definition of a protocol that allows you not to get in each other's way. Collaboration....means amplification. The amplification you get by connecting up a bunch of human beings who are listening to each other, interested in each other, bring separate depth to the problem, bring breadth that gives them interest in the entire solution, allows them to communicate on multiple different levels, verbally, in writing, in feeling, in acting, in pictures" (Nelson 2008).

Although less easy to quantify than the number of authors or affiliations on a paper, a closer look at many laboratories indicates that collaborative research efforts-contrary to what their names imply - usually involve cooperation, not collaboration. Many of us have encountered the situation where scientists who work as part of a group on a specific aspect of a research project can explain their tasks very well, but are unable to comment on the overall strategy or on how the contributions of others fit into the project. Even worse, people frequently refuse to share their ideas or unpublished results with others, not only with outsiders but even with those of their own group. When Theodore H. Bullock, one of the leading comparative neurobiologists of the twentieth century, and famous for his attitude of open exchange of ideas and results (for obituaries, see Zupanc 2006; Zupanc and Zupanc 2008; Barth 2008), and I once talked about such encounters, he called these laboratories "research factories". Indeed, the way research is conducted in such labs resembles the manufacturing of an industrial product along an assembly line.

The decision made by many principal investigators to opt for a cooperative strategy instead of a collaborative one is not surprising in times when funding rates in many countries are at historic lows, and thus competition is fiercer than ever. Cooperation by employing highly skilled specialists who focus on separate, narrowly defined areas of expertise is regarded as an efficient way to maximize research output in terms of the number of papers published. Moreover, many investigators view this approach of compartmentalization of tasks as the only model possible to carry out interdisciplinary projects. By contrast, collaboration may not only be more time-consuming but also requires, in addition to the need to have adequate research skills in place, the cultivation of a work environment that promotes social interactions. Yet it is this socially receptive culture that creates a learning community through which the individual members synergistically share their ideas and jointly develop new ideas, thus enabling each of them to grow intellectually and professionally.

\section{William T. Keeton: an attitude of collaboration, and the story behind an article}

In such times of extremely high levels of competition in the world of science it is particularly important to be reminded of the collaborative spirit's intrinsic value. The article by Jonathan Hagstrum and Geoffrey Manley in this issue (Hagstrum and Manley 2015) is the direct result of such a spirit. This would hardly be worth an editorial had the investigator, William ('Bill') T. Keeton, who created and promoted this culture of openly sharing ideas with others and encouraging collaborations, not passed away a long time ago. When Keeton died in 1980, at the age of just 47, he left behind not only an exceptional legacy of research creativity and productivity, but also an immense set of both published and unpublished original data. It is part of these data on which the study by Hagstrum and Manley is based -35 years after Keeton's death. 


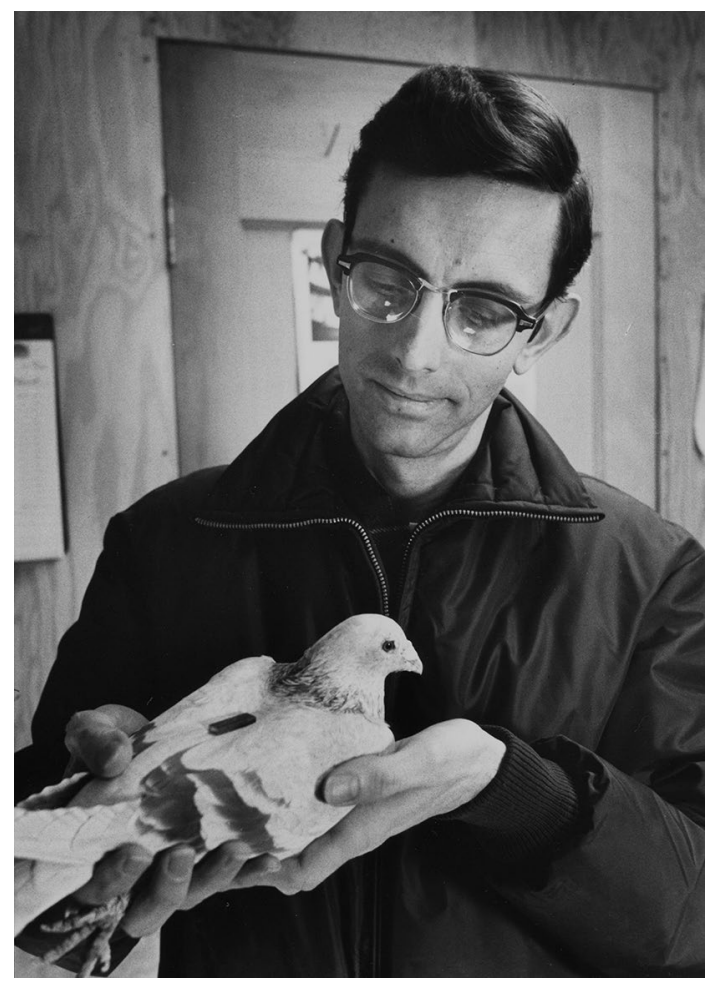

Fig. 2 William T. Keeton with a Cornell pigeon. Photographer unknown. Courtesy Charles Walcott/Cornell University

Just like the story behind this article, Bill Keeton (Fig. 2) was unusual in many ways [for an obituary, see (Emlen 1981)]. In the same year (1958) that Keeton received his Ph.D., he joined the faculty of the Department of Entomology at Cornell University (Ithaca, New York) to work on millipede systematics. In 1965, he moved to the new Department of Neurobiology and Behavior that he had helped to create, and there, 1 year later, started a vigorous research program on pigeon homing. This program ran successfully over the following 14 years until his death and involved not only resident staff, but also many graduate students and some 60 undergraduates who assisted with the experiments. During that time, he also interacted closely with a second faculty member who worked on avian migration, Stephen T. Emlen. In addition, he attracted numerous visitors from the US and abroad, so that Ithaca became a world-renowned center of research into homing and navigation in birds. Timothy Larkin, a research technician at that time, remembers Keeton's approach to collaborate with visitors: "Scientists came here not only because they could find experimental animals in an abundance that few could match, but also because Bill let them do their own research, not his...his attitude was to be a 'midwife' to someone else's creativity" (T. S. Larkin, personal communication). In some years, as many as 1500 pigeons of different strains, ages, and levels of experience were available for experiments. These birds were used for multiple approaches to study the mechanisms of homing, such as the role of the sun compass in orientation, the possible existence of navigational maps, and the effects of various sensory cues on pigeon homing. His findings and those of his students led to a paradigm shift in orientation research. Instead of attempting to explain homing behavior by a single-cue theory, he emphasized the redundancy of mechanisms, involving multi-sensory cues. After Keeton's death, under the leadership of Stephen Emlen, several of his former associates organized and indexed the results of 2500 such experiments (Brown et al. 1983). The William T. Keeton Database, which includes both published and unpublished data, is available to the scientific community from Dr. Charles Walcott at Cornell University (e-mail: cw38@ cornell.edu).

Among the visitors whose experiments are recorded in the database was Geoffrey Manley, a researcher in comparative hearing mechanisms, then at McGill University in Canada. He had suggested the cochlea as an organ that senses the magnetic field because during flight it is oriented at $90^{\circ}$ to the flight path and has a steady current flowing from endolymph to perilymph. To test this hypothesis, he collaborated with Keeton during visits to Cornell in 1973, 1974, and 1975, using 200 de-cochleated Cornell pigeons. These birds were intended to be released under overcast skies to exclude their sun-compass orientation. Unfortunately, the weeks following the operations lacked total overcast and, since the birds had to be exercised daily, Keeton eventually concluded that redundant cues had made the releases of these birds uninterpretable. However, although originally designed to verify a different hypothesis, these data have provided the opportunity to relate them to atmospheric profiles recorded and archived by the National Oceanic and Atmospheric Administration, to examine whether pigeons use infrasound for orientation during homing. The results of this analysis are presented in the Hagstrum and Manley paper in the present issue. In an accompanying review, Robert C. Beason and Wolfgang Wiltschko discuss these results within the broader context of pigeon orientation, and remind us of the important questions that still remain unanswered (Beason and Wiltschko 2015).

Bill Keeton's willingness to share his ideas and unpublished results with others was legendary and did not end just with peers he agreed with on scientific questions. When a group of researchers at the University of Pisa, Italy, proposed that homing pigeons use olfactory cues to home from unfamiliar sites (Papi et al. 1972), Keeton and his colleagues tried to test the olfactory hypothesis, but were unable to convince themselves of its universal applicability (Keeton 1974; Keeton and Brown 1976; Keeton et al. 1977; Hermayer and Keeton 1979). He, therefore, invited Papi and part of his group to Ithaca to resolve the issue through 
collaborative experiments. In the resulting article published in the Journal of Comparative Physiology A, Keeton, Papi, and their associates jointly presented their results, but still could not agree on the final interpretation. They, therefore, did what may well be unmatched in the entire scientific literature: they included two separate discussion sections in the paper, one written by the Italian authors and the other by the Americans (Papi et al. 1978).

Keeton was famous not only for his collaborative spirit shown toward colleagues, but soon after joining the faculty of Cornell he also gained a reputation as a superior and highly dedicated teacher. His engaging teaching style left a lasting impression on myriads of students, so that the introductory biology course he taught was known around campus simply as the 'Keeton Course'. Based on this course, he devoted 5 years of his life to writing Biological Science-a decision in line with his attitude toward sharing knowledge and serving the community. This text integrates the various disciplines of biology into a single book, and relates them to the underlying principles of evolution (Keeton 1967). Keeton's textbook, which appeared in several editions, has had a major influence on the teaching of biology in North America, and introduced untold numbers of students to the field.

The fact that data from the experiments Keeton and his collaborators conducted are still being published 35 years after his death, and that the results and the interpretation reported in this issue are certain to stimulate further discussions, likely to trigger controversy, but hopefully will initiate fruitful interactions among the readers of our journal, is testament to the power of one such collaborative spirit-in the past as well as in the future.

Acknowledgments I am indebted to Robert C. Beason, Jonathan T. Hagstrum, Timothy S. Larkin, Geoffrey A. Manley, Charles Walcott, Frederick B. Zupanc, and Marianne M. Zupanc for their comments on previous versions of the manuscript.

Note added in proof Our own capability to collaborate was sadly put to the test shortly after Robert C. Beason, whom I had invited to discuss the research article by Hagstrum and Manley within the broader context of pigeon orientation, submitted his manuscript. Due to a severe accident, he became unable to revise his paper. In this difficult situation, Wolfgang Wiltschko agreed to perform the revision and to add his own perspective to the discussion. The resulting review article is published under joint authorship in this issue of the Journal of Comparative Physiology A (Beason and Wiltschko 2015).

\section{References}

Barth FG (2008) Early recollections of one in a crowd of admirers. J Comp Physiol A 194:115-117

Beason RC, Wiltschko W (2015) Cues indicating location in pigeon navigation. J Comp Physiol A. doi:10.1007/s00359-015-1027-2

Brown I, Larkin T, Emlen ST (1983) Fourteen years of pigeon homing data. Auk 101:622-623

Emlen ST (1981) In memoriam: William T. Keeton. The Auk 98:167-172

Hagstrum JT, Manley GA (2015) Releases of surgically deafened homing pigeons indicate that aural cues play a significant role in their navigational system. J Comp Phsyiol A. doi:10.1007/ s00359-015-1026-3

Hermayer KL, Keeton WT (1979) Homing behavior of pigeons subjected to bilateral olfactory nerve section. Monitore Zool Ital (NS) 13:303-313

Keeton WT (1967) Biological science. W.W. Norton \& Company, New York

Keeton WT (1974) Pigeon homing: no influence of outward-journey detours on initial orientation. Monitore Zool Ital (NS) 8:227-234

Keeton WT, Brown AI (1976) Homing behavior of pigeons not disturbed by application of an olfactory stimulus. J Comp Physiol A 105:252-266

Keeton WT, Kreithen ML, Hermayer KL (1977) Orientation of pigeons deprived of olfaction by nasal tubes. J Comp Physiol A 114:289-299

Nelson R (2008) Learning and working in the collaborative age. Speech given on April 11, 2008 at the Apple Education Leadership Summit. Available from http://www.edutopia.org/randynelson-school-to-career-video. Accessed on 8 Apr 2015

Papi F, Fiore L, Fiaschi V, Benvenuto S (1972) Olfaction and homing in pigeons. Monitore Zool Ital (NS) 6:85-95

Papi F, Keeton WT, Brown AI, Benvenuti S (1978) Do American and Italian pigeons rely on different homing mechanisms? J Comp Physiol A 128:303-317

Roschelle J, Teasley SD (1995) The construction of shared knowledge in collaborative problem solving. In: O'Malley C (ed) Computer supported collaborative learning. Springer, Berlin/Heidelberg, pp 69-97

Zupanc GKH (2006) Theodore H. Bullock: trailblazer in neurobiology. Nature 439:280

Zupanc GKH, Zupanc MM (2008) Theodore H. Bullock: pioneer of integrative and comparative neurobiology. J Comp Physiol A 194:119-134 